\title{
Free learning as a function of meaningfulness and encoding cues
}

\author{
JAMES H. CROUSE \\ STATE UNIIERSITY OF NEW YORK AT BINGHAMTON
}

Two experiments were performed in which cncoding cues were presented with items of low or high meaningfulness in free learning. When the lists varied widely in meaningfulness, encoding cues uere found to facilitate the lou meaningfulness items but to decrease slightly learning of the high meaningfulness items.

The number of implicit associational responses (LARs) elicited by a verbal item (Underwood, 1965) varies directly with its meaningfulness $(M)$. An understanding of the effects of $M$ on free learning may be partly achieved by determining the role played by IARs. If $S$ can make an IAR that is easier to recall than the presented item, he may encode the presented item via the IAR during learning.

A LM item like $m y t$ will elicit few IARs offering little chance for encoding; therefore if $S$ is aided in making an appropriate IAR by presentation of an encoding cue (e.g., myth), encoding and subsequent recall may be increased. However, when a HM item like $y e l$ is presented, it is likely to elicit a variety of IARs like "yellow," "yelp," or "yell," which S could use to encode the item. Therefore, presentation of an encoding cue for a single IAR may not be as efficient an encoding aid as the IARs that would normally occur. Thus, presentation of an encoding cue may decrease recall of a HM item. Evidence for this interpretation in short-term memory has been obtained by Lindley (1963, 1965, Experiment II) who found an inverse relationship between $M$ and facilitation due to encoding cues. The present experiments study the effects of $M$ and encoding cues in free learning.

\section{Method}

\section{EXPERIMENT I}

Twenty Ss from the Virginia Military Institute were assigned to each of the six conditions of a 2 by 3 factorial design. Each $S$ learned a single 16-item list of CVC trigrams. The first variable was the $\mathbf{M}$ of the items, either LM (mean association value of 27.63, Archer, 1960) or HM (mean association value of 84.93 ). The second variable was encoding cues. Cues were either presented with instructions to use them in learning the trigrams (CI), e.g., $S$ was presented juv followed by juvenile and was told the cue would help him recill the trigram, or cues were presented with no instructions to use them (CNI), e.g., $S$ was presented jul followed by julchilc, but was not instructed to use the cue; or no cues were presented (NC), e.g., $\mathrm{S}$ was presented only jul. For some trigrams, the first three letters of the trigram were the same as those of the cue word, and for other trigrams the first two letters of the trigrams were the same as the cue word and the third letter was different. The mean L-count frequency (Thorndike \& Lorge, 1944) of the cue words in the LM and HM conditions was 160.56 and 214.68 , respectively.

The items were read by $E$ at a 5 -sec. rate. In the CI and CNI conditions each trigram was spelled and word pronounced twice during its 5-sec. interval (e.g., "j-u-v juvenile," "j-u-v juvenile"'). In the NC condition each trigram was spelled twice during its 5-sec. interval. Two $\mathrm{min}$. written recall was given after each trial in which $S$ was to recall the trigrams. Three trials were given with a different presentation order on each. The Ss were run in small groups of one to five which were randomly assigned to the six conditions.

\section{Results and Discussion}

An anclysis of variance on the number of correct responses revealed a highly significant effect of trials as expected, $F=467.85$, $d f=2 / 114$. Since none of the interactions with trials was significant, the average number of correct responses on the three trials was computed for each $S$ and these group means were chosen for presentation in Table 1. The variable of encoding cues had a significant effect, $F=7.30, \mathrm{df}=$ $2 / 114, p<.01$. Individual comparisons indicated that acquisition did not differ significantly in the $\mathrm{CI}$ and CNI conditions, $F<1.0, \mathrm{df}=1 / 114$, but the $\mathrm{CI}$ condition was higher than the NC condition as was the CNI condition, $F s=13.05$ and 8.31 , respectively, $\mathrm{df}=1 / 114$, ps $<.01$. Acquisition was numerically higher in the HM than LM condition, but this difference only approached significance, $F=3.34, \mathrm{df}=1 / 114$. The effect of primary interest was the $\mathbf{M}$ by Cues interaction, and this effect was not significant, $F=2.50, d f=2 / 114$.

The expected interaction showing that cues facilitated free learning of LM items and had less effect or interfered with acquisition of $\mathrm{HM}$ items was not obtained. One reason may have been that the HM items did not have high enough $M$. The HM items were not learned significantly faster than the LM items and may not have elicited a great enough number of IARs to provide a more efficient encoding source than the single cues provided by $\mathrm{E}$. This suggests that a wider

\begin{tabular}{lcccc}
\multicolumn{5}{c}{ Table 1. Mean number of correct responses per trial } \\
& \multicolumn{2}{c}{ Experiment I } & \multicolumn{2}{c}{ Experiment II } \\
& $\mathrm{LM}$ & $\mathrm{HM}$ & $\mathrm{LM}$ & $\mathrm{HM}$ \\
$\mathrm{Cl}$ & 9.91 & 10.31 & 10.51 & 11.43 \\
$\mathrm{CNI}$ & 9.31 & 10.20 &.- & $\cdots$ \\
$\mathrm{NC}$ & 7.83 & 3.80 & 7.64 & 12.66
\end{tabular}


range of $\mathbf{M}$ would lead to the expected $\mathbf{M}$ by Cues interaction. Experiment II tests this hypothesis by using very $L M$ trigrams and $H M$ words.

\section{Method}

\section{EXPERIMENT II}

Fifteen Ss from Harpur College were assigned to each of the four cells of a 2 by 2 factorial design in which the two variables were $M, L M$ vs. HM, and encoding cues, CI vs. NC. The method was the same as Experiment I except for the following: (1) The LM list was constructed of trigrams having a mean summed letter association value of 19.37 (Underwood \& Schulz, $1960)$, and the HM list consisted of three letter words in which the mean was 46.87 . A variety of consonantvowel-consonant sequences was used for the items in both lists, e.g., CVC, VCC, CCC, CCV for LM and CVC, VVC, VCV, VCC for HM. (2) The mean L-count frequency of the cue words in the CI condition for LM and HM was 30.31 and 28.62, respectively. The first three letters of the cue word always were the same as the item the cue word followed. (3) A 1 1/2 min. written recall period was given after each trial. Resulis

An analysis of variance on the number of correct responses revealed a significant trials effect, $F=206.21$, $\mathrm{df}=2 / 112, \mathrm{p}<.01$, but since none of the interactions with trials was significant, the mean number of correct responses on the three trials was computed for each $S$ and these group means are presented in Table 1. Acquisition was greater with $\mathrm{HM}$ than $\mathrm{LM}, \mathrm{F}=\mathbf{2 8 . 9 9}$, $\mathrm{df}=1 / 56, \mathrm{p}<.01$, and the $\mathrm{Cl}$ condition was numerically superior, but not significantly so, to the NC condition, $\mathrm{F}=2.29, \mathrm{df}=1 / 56$. The expected interaction between $M$ by Cues was obtained, $F=13.17, \mathrm{df}=1 / 56, \mathrm{p}<.01$, with cues increasing learning with LM but slightly decreasing learning in the HM condition.

\section{Discussion}

The present experiments show that encoding cues, as compared with no encoding cues, facilitated free learning of both LM and HM trigrams (Experiment I), but when a wider range of $M$ was employed (Experiment II), encoding cues tended to increase LM acquisition and decrease HM acquisition. Essentially these same results have been found in short-term memory for individual items by Lindley (1963) who found that encoding cues increased recall of LM trigrams but slightly decreased recall of three letter words.

The present results are consistent with the hypothesis that Ss try to integrate items via IARs during free learning. If this hypothesis is true, then it seems reasonable to expect that providing $S$ with an encoding cue should have an effect on learning that is inversely related to the number of IARs elicited by the items. Presenting $S$ with an encoding cue will facilitate learning when $S$ has difficulty making an appropriate IAR for encoding. On the other hand, when $S$ is easily able to make the appropriate IARs, then presenting him with a single encoding cue may not be as efficient an encoding aid as his own generated IARs.

\section{References}

Archer, E. J. Re-evaluation of the meaningfulness of all possible CVC trigrams. Psychol. Monogr., 1960, 74, No. 10 (Whole No. 497).

Lindley, R. H. Effects of controlled coding cues in short-term memory. J. exp. Psychol., 1963, 66, 580-587.

Lindley, R. H. Effects of trigram-recoding cue complexity on shortterm memory. J. verbal Learn. Verbal Behav., 1965, 4, 274-279.

Thomdike, E. L., \& Lorge, I. The teachers word book of 30,000 words. New York: Columbia University Press, 1944.

Underwood, B. J. False recognition produced by implicit verbal responses. J. exp. Psychol., 1965, 70, 122-129.

Underwood, B. J., \& Schulz, R. W. Meaningfulness and rerbal learning. Philadelphia: Lippincott, 1960. 